Brit. J. vener. Dis. (1958), 34, 166.

\title{
CANDIDA ALBICANS URETHRITIS* REPORT OF A CASE
}

BY

\author{
W. FOWLER
}

The Royal Hospital, Wolverhampton

A case of Candida albicans urethritis is thought to be worth recording as very few similar reports have appeared in the literature.

\section{Case Report}

A married man, 54 years of age, attended the clinic on October 17, 1955, complaining of urethral discharge, constant urethral irritation, and slight frequency of micturition of 3 days' duration. He had been in good health until the onset of these symptoms. Extra-marital coitus was denied. There was a very scanty, thin, greyish, mucopurulent urethral discharge. The glans penis and prepuce were normal. The prostate felt normal. The three-glass urine test showed:

Glass 1 urine hazy with many thick, white, heavy threads; Glass 2 urine clear except for a few threads;

Glass 3 urine clear.

* Received for publication 18 March, 1958.
Anterior Urethroscopy.-Only the smallest cannula (18F) could be introduced into the urethra and then only to the mid-point of the anterior urethra. The distal half of the urethra was rigid and did not inflate with air. The epithelium here was white and thickened and readily fissured longitudinally. It is worth mentioning that, although the cannula was well lubricated, its passage along the urethra imparted a gritty sensation similar to that obtained by pulling one piece of sand-paper across another.

The stricture at the mid-point of the anterior urethra disappeared with air inflation. Beyond this point the urethra presented a most bizarre appearance (Figure). The surface was studded with solid-looking white excrescences which protruded far into the lumen and appeared to be firmly fixed to the mucosa. Indeed, these patches of membrane were only detached with difficulty. Their removal left no bleeding points, the mucous membrane beween them was normal, and the wall responded readily to inflation.

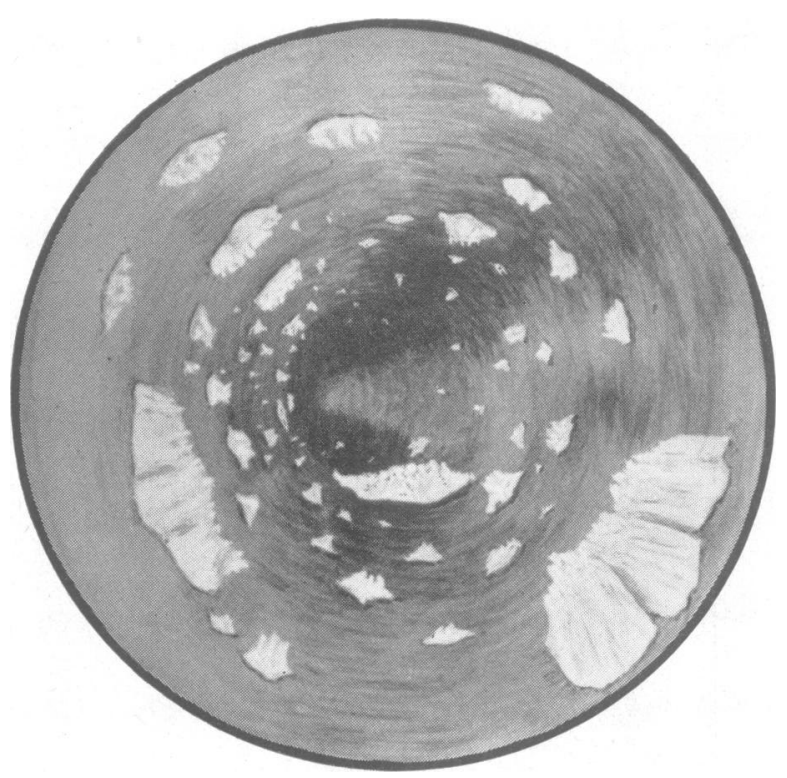

Figure.-Drawing of urethra showing patches of membrane. 
Laboratory Findings.-Microscopically the urethral discharge and the urinary threads consisted of masses of mycelia and spores typical of Candida albicans, many large epithelial cells, a few pus cells, and a very few extracellular Gram-negative cocco-bacilli. On Sabouraud's medium, the urethral discharge produced a profuse growth of Candida albicans. Sub-cultures on corn meal agar confirmed the nature of the fungus. Chocolate agar cultures of the discharge were negative for gonococci.

The urine contained over 2 per cent. glucose by the clinitest, and the fasting blood sugar was $350 \mathrm{mg}$. per cent.

Diagnosis.-In this case the diagnosis of Candida albicans urethritis seemed to be completely established. The urethroscopic findings suggested that the infection had penetrated to the sub-mucosa in the distal half of the antcrior urethra but was superficial in the proximal half. The spasm at the junction of these parts was due presumably to the irritative effect of the sub-mucosal infiltratration on normal muscle. There were no symptoms indicative of posterior urethral infection, but this point could not be determined exactly as the narrowness of the anterior urethra precluded posterior urethroscopy. It was impossible to determine if there was any sub-clinical involvement of the prostate. The three-glass test showed that the infection was limited to the lower urinary tract.

Treatment and Progress.-The patient was seen at least three times weekly until diabetic treatment was started, and then once weekly until December 5, 1955, after which he was not seen again until March, 1956. At each visit any urethral discharge or urinary deposit present was examined for fungi. Urethroscopy was carried out at weekly intervals.

$9 \mathrm{ml}$. diamidophenylamine dihydrochloride $(\mathrm{M}$ and B 938) were available, and $3 \mathrm{ml}$. were instilled into the urethra on three successive days before diabetic treatment commenced. This treatment produced a slight fleeting sensation of warmth in the urethra. Four days after the last instillation heavy threads were no longer present in the urine and on urethroscopy the proximal half of the urethra was found to be free from membrane but the mucosa here was now slightly inflamed. The distal portion of the urethra was unchanged. No further local treatment was given as it was thought unlikely that such treatment could have any effect on the sub-mucous infection.

The urethral irritation now lessened in intensity and was present only intermittently. The urethral discharge became even more scanty and the urine became clear except for a few threads. Microscopically the urethral discharge contained very few filaments although spores were still very plentiful. The erythema quickly disappeared from the proximal part of the urethra.

Thus considerable improvement had occurred before diabetic treatment was started on November 1, 1955. By November 17 the diabetes was controlled; there was no discharge from the urethra and the urine was clear except for a few light threads. The centrifuged urinary deposit contained a few spores. On November 28 the urine was normal macroscopically and microscopically.
The pathological changes in the distal part of the anterior urethra disappeared slowly and the urethra did not return to normal until December 5, 1955; 3 months later there were no clinical signs of urethritis and the urethra was completely normal on urethroscopic examination.

The patient's wife was examined several times. There were no clinical signs of vaginal infection. Initially there were a few yeast spores in the vaginal secretion but these soon disappeared without treatment.

\section{Comment}

The incidence of Candida albicans urethritis is difficult to ascertain but appears to be very low. The present case is from a series of 750 consecutive cases of non-specific urethritis, but the actual incidence must be very much lower than this. Harkness (1950) appears to have seen only one case and in a review of the literature mentions no more than thirteen cases. I have not seen the condition before nor have I seen any other case of urethritis in which masses of mycelia were seen in urethral films or in which fungal elements were present in films taken after careful cleansing around and inside the meatus. Although I have carried out urethroscopy as a routine examination in non-specific urethritis for many years, I have never before seen patches of membrane in the urethra.

Harkness (1950) says that fungi seldom attack the normal urethra and quotes experimental work to show that urethral trauma predisposes to the infection. He also wonders if devitalization of the urethra by diabetes is a predisposing factor, as the condition often occurs in association with this metabolic disease. This may be so, but it should be noted that Candida albicans urethritis is so rare in diabetes that physicians with long experience of the latter disease tell me that they have yet to see this type of urethritis.

The source of the infection is uncertain and the present case offers no help towards solving the problem. Little significance can be attached to finding fungal elements in the vaginal secretion of the consort of the patient as the habitat of the fungus is such that the urethral infection could well have an endogenous origin.

From the comprehensive review of Harkness (1950) it would appear that Candida albicans urethritis has no distinctive symptoms or signs other than patches of membrane in the urethra, and it is not known if membrane formation is a constant feature of the infection although positive urethroscopic findings have been recorded in the few cases in which this examination was carried out while the disease was active. In these cases the urethroscopic 
appearances were seldom so striking as in the case reported above and usually only a single plaque of membrane was seen.

A complaint of constant urethral irritation is not very common in urethritis. In the present case this symptom was due to the patches of membrane and if membrane is always produced constant urethral irritation may be a characteristic feature of the infection.

The preponderance of large epithelial cells in the urethral discharge of my case may also be characteristic of a Candida albicans infection, as this desquamative type of discharge is common in vaginal thrush.

I was unable to discover the extent of involvement of the lower urinary tract, but it is known that the infection can invade the posterior urethra, and Coutts (1948) has recorded prostatic involvement.

Discussing diagnosis, Harkness (1950) says that this is only warranted when spores with or without mycelial elements have been shown to be constantly present in films of the urethral discharge on repeated examination. I believe that the presence of fungal elements and especially of mycelia in urethral films taken after careful cleansing of the urinary meatus is an indication for urethroscopy. If membrane is observed in the urethra, the diagnosis is certain, and all that remains is to culture the discharge on Sabouraud's or some other special medium for the proper identification of the fungus.
In the absence of corroborative urethroscopic evidence, the diagnosis is more difficult and will depend not only upon the persistence of fungi in the urethral discharge but also on failure to discover any other cause for the urethritis.

The present case and some of the cases described by Harkness (1950) show that Candida albicans urethritis tends to be self-limiting especially after any associated diabetes has been brought under control. This being so, it is difficult to say whether the urethral instillations were responsible for the early response in my patient or whether the cures reported in cases given other forms of local treatment were due to these treatments. However, it seems reasonable to assume that where the infection is superficial local treatment with one of the effective fungicides now becoming available may shorten the duration of the illness, and that a similar result may be obtained following systemic treatment with pentamide or hydroxystilbamidine where the infection has penetrated to the urethral submucosa.

I am extremely grateful to Mr. Joseph Plant, S.R.N., former assistant tutor at the Royal Hospital, for his faithful drawing of the urethroscopic appearance.

\section{REFERENCES}

Harkness, A. H. (1950). “Non-gonococcal Urethritis”, pp. 248-254, Livingstone, Edinburgh.

Coutts, W. E. (1948). Brit. J. vener. Dis., 24, 109. 